\title{
The optical design concept of SPICA-Safari
}

\author{
Willem Jellema $^{* a, b}$, Bob Kruizinga ${ }^{c}$, Huib Visser ${ }^{c}$, Teun van den Dool ${ }^{\mathrm{d}}$, Carmen Pastor Santos ${ }^{\mathrm{e}}$, \\ Josefina Torres Redondo ${ }^{\mathrm{f}}$, Martin Eggens ${ }^{\mathrm{a}}$, Marc Ferlet ${ }^{\mathrm{g}}$, Bruce Swinyard ${ }^{\mathrm{g}}$, Kjetil Dohlen ${ }^{\mathrm{h}}$, Doug \\ Griffin $^{\mathrm{g}}$, Luis Miguel Gonzalez Fernandez ${ }^{\mathrm{e}}$, Tomas Belenguer ${ }^{\mathrm{e}}$, Hideo Matsuhara ${ }^{\mathrm{i}}$, Mitsunobu \\ Kawada ${ }^{\mathrm{i}}$ and Yasuo Doi ${ }^{\mathrm{j}}$ \\ ${ }^{a}$ SRON Netherlands Institute for Space Research, Department of Low Energy Astrophysics, P.O. \\ Box 800, 9700 AV Groningen, the Netherlands; ${ }^{b}$ Kapteyn Astronomical Institute, University of \\ Groningen, P.O. Box 800, 9700 AV Groningen, the Netherlands; ${ }^{\mathrm{c}}$ TNO Optics, P.O. Box 155, 2600 \\ AD Delft, the Netherlands; ${ }^{\mathrm{d}}$ TNO Optomechatronics, P.O. Box 155, 2600 AD Delft, the \\ Netherlands; ${ }^{\circ}$ Instituto Nacional de Técnica Aeroespacial (INTA), Carretera de Ajalvir Km 4.5, \\ 28850 Torrejón de Ardoz, Madrid, Spain; ${ }^{\mathrm{f} C e n t r o ~ d e ~ A s t r o b i o l o g i a ~(I N T A-C S I C), ~ C a r r e t e r a ~ d e ~}$ \\ Ajalvir Km 4.5, 28850 Torrejón de Ardoz, Madrid, Spain; ${ }^{g}$ SFTC-RAL Space, Harwell Science \& \\ Innovation Campus, Didcot, OX11 0QX, United Kingdom; \\ ${ }^{\mathrm{h}}$ Laboratoire d'Astrophysique de Marseille, UMR6110, CNRS/Université de Provence, 38 rue \\ Frédéric Joliot Curie, 13388 Marseille Cedex 13, France; ${ }^{i} J a p a n$ Aerospace Exploration Agency \\ (JAXA), Institute of Space and Astronautical Science (ISAS), Department of Infrared Astrophysics, \\ 3-1-1 Yoshinodai, Chuo-ku, Sagamihara, Kanagawa 252-5210, Japan; ${ }^{j}$ The University of Tokyo, \\ Graduate School of Arts and Sciences, Department of Earth Science and Astronomy, 3-8-1 Komaba, \\ Meguro-ku, Tokyo 153-8902 Japan
}

\begin{abstract}
The Safari instrument on the Japanese SPICA mission is a zodiacal background limited imaging spectrometer offering a photometric imaging $(\mathrm{R} \approx 2)$, and a low $(\mathrm{R}=100)$ and medium spectral resolution $(\mathrm{R}=2000$ at $100 \mu \mathrm{m})$ spectroscopy mode in three photometric bands covering the 34-210 $\mu \mathrm{m}$ wavelength range. The instrument utilizes Nyquist sampled filled arrays of very sensitive TES detectors providing a 2'x2' instantaneous field of view. The all-reflective optical system of Safari is highly modular and consists of an input optics module containing the entrance shutter, a calibration source and a pair of filter wheels, followed by an interferometer and finally the camera bay optics accommodating the focal-plane arrays. The optical design is largely driven and constrained by volume inviting for a compact threedimensional arrangement of the interferometer and camera bay optics without compromising the optical performance requirements associated with a diffraction- and background-limited spectroscopic imaging instrument. Central to the optics we present a flexible and compact non-polarizing Mach-Zehnder interferometer layout, with dual input and output ports, employing a novel FTS scan mechanism based on magnetic bearings and a linear motor. In this paper we discuss the conceptual design of the focal-plane optics and describe how we implement the optical instrument functions, define the photometric bands, deal with straylight control, diffraction and thermal emission in the long-wavelength limit and interface to the large-format FPA arrays at one end and the SPICA telescope assembly at the other end.
\end{abstract}

Keywords: SPICA, Safari, far-infrared, spectroscopy, imaging spectrometer, optics, Mach-Zehnder, interferometer

\section{INTRODUCTION}

The Japanese mission SPICA led by JAXA is anticipated to become the next great leap forward in far-infrared astronomy. With an expected launch in the early 2020s, SPICA will fill the gap between Herschel, ALMA and JWST, featuring a cryogenic $3 \mathrm{~m}$ class telescope opening up the scientific window of zodiacal background limited observations in the far-infrared[1].

*W.Jellema@sron.nl; phone +31-(0)50-3634058; fax +31-(0)50-363-4033; http://www.sron.nl

Space Telescopes and Instrumentation 2012: Optical, Infrared, and Millimeter Wave, edited by Mark C. Clampin, Giovanni G. Fazio, Howard A. MacEwen, Jacobus M. Oschmann, Jr., Proc. of SPIE Vol. 8442, 84420S

(C) 2012 SPIE · CCC code: $0277-786 / 12 / \$ 18 \cdot$ doi: $10.1117 / 12.927153$ 
Making full advantage of a deeply cooled telescope $(<6 \mathrm{~K})$ the Safari instrument on SPICA is a highly sensitive widefield imaging photometer and spectrometer operating in the 34-210 $\mu \mathrm{m}$ wavelength range. Utilizing Nyquist-sampled filled arrays of very sensitive Transition Edge Sensors (TES), Safari offers a photometric imaging $(\mathrm{R} \approx 2)$, and a low (R $=100)$ and medium resolution $(\mathrm{R}=2000$ at $100 \mu \mathrm{m})$ imaging spectroscopy mode in three photometric bands within a 2'x2' instantaneous Field of View (FoV).

Like the SPIRE instrument on Herschel, Safari utilizes an imaging Fourier Transform Spectrometer (FTS). An FTS is seen to best address the scientific need for large-area, wide-band spectroscopic mapping enabling blind, wide-field spectroscopic surveys to characterize the chemistry and dynamics of many sources. The combination of very sensitive large-format filled TES arrays fully sampling a large instantaneous FoV and its high spectral resolving power, makes Safari an extremely powerful unbiased spectroscopic imaging instrument characterizing for example the spatial and spectral energy distribution of very faint point sources just above the natural background. An overview of the key scientific themes to be addressed by Safari and the instrument design is given in [2-3].

Inspired by the optical layout of Herschel-SPIRE [4-5] an initial optical design was made at RAL during Phase A1 of the project still under UK leadership back in 2009. This optical design incorporated a Mach-Zehnder interferometer in a Cat's Eye configuration. The FTS concept builds on a mechanism developed by TNO for the Darwin mission, which is based on magnetic bearings providing high precision translation at very low cryogenic dissipation[6-7].

In 2010 it was decided to select a smaller sized launcher. Consequently the SPICA Telescope Assembly (STA) had to be rescoped. In order to fit the STA into a tighter fairing (5S), the entrance pupil diameter of the telescope was reduced from $3.4 \mathrm{~m}$ to $3.0 \mathrm{~m}$ in addition to general spacecraft redesign. It became evident that the existing concept for the focal plane optics would no longer be compatible with the changed spacecraft environment. In parallel the design of the TES arrays had significantly evolved and matured, leading to additional requirements and boundary conditions not envisaged during the Phase A1 study

In what follows we will describe the design evolution from the Phase A1 status to the present configuration. We first summarize the main external constraints and boundary conditions imposed by the SPICA spacecraft and telescope. Next we shortly discuss the design goals and key requirements applicable to the optical design. The conceptual optical design is described addressing the implementation of the optical functions and the way we deal with controlling straylight and diffraction. The paper is concluded by an outlook on future work.

\section{EXTERNAL CONSTRAINTS AND BOUNDARY CONDITIONS}

\subsection{The SPICA telescope interface}

One of the major changes relative to the Phase A1 study in 2009 was a telescope redesign towards an entrance pupil diameter of $3.0 \mathrm{~m}$ instead of $3.4 \mathrm{~m}$. Although the final telescope interface is currently not yet frozen, an intermediate telescope design proposed by ESA as input for an industrial study was assumed to update the Safari optical design. The main characteristics of the telescope design assumed in this paper are given in Table 1. In order to fit the telescope into the smaller fairing the distance between M1 and M2 was decreased and the effective focal length was reduced. The corresponding $\mathrm{f} / \#$ changed from $\mathrm{f} / 6 \mathrm{fo} \mathrm{f} / 5.4$ and the focal plane radius of curvature decreased to about $700 \mathrm{~mm}$. Because of the smaller $\mathrm{f} / \#$ and effective focal length of the telescope the plate scale in the focal plane was significantly reduced and opto-mechanical interference of the pick-off mirror structures of the Focal Plane Instruments (FPI's) became problematic. For that reasons the back-focal length was adjusted as well as the relative position of the telescope with respect to the mounting plane on the optical bench allowing the instrument to move away from the optical axis.

Table 1. Telescope parameters assumed for the Safari optical design.

\begin{tabular}{|l|l|}
\hline Parameter & Value \\
\hline Pupil stop position & on M2 \\
\hline Effective focal length (EFL) & $16200 \mathrm{~mm}$ \\
\hline
\end{tabular}




\begin{tabular}{|l|l|}
\hline Back focal length (BFL) & $898 \mathrm{~mm}$ \\
\hline $\mathrm{M} 1-\mathrm{M} 2$ distance & $2511 \mathrm{~mm}$ \\
\hline Focal ratio (f/\#) & 5.4 \\
\hline Entrance pupil diameter (EPD) & $3000 \mathrm{~mm}$ \\
\hline Focal plane curvature & $709.3 \mathrm{~mm}$ \\
\hline $\mathrm{R}_{1}$ radius of curvature M1 & $-6360.4456 \mathrm{~mm}$ \\
\hline $\mathrm{K}_{1}$ conic constant M1 & -1.020833 \\
\hline $\mathrm{R}_{2}$ radius of curvature M2 & $-1665.3755 \mathrm{~mm}$ \\
\hline $\mathrm{K}_{2}$ conic constant $\mathrm{M} 2$ & -2.406615 \\
\hline
\end{tabular}

The Safari instrument picks off from the telescope focal plane at an off-axis position of 7' with a science FoV of 2'x2'. During the Phase A1 study it was found that Safari would not be able not pick-off further off-axis without introducing significant field aberrations due to uncompensated focal plane curvature.

\subsection{Instrument optical bench (IOB)}

As part of the spacecraft redesign the original circular optical bench of a composite material was changed into an aluminum triangular shaped body (a box with walls and a cover) mono-statically mounted to the telescope mechanical interface. This change significantly decreased the available volume for the focal plane instruments. After several design iterations between ESA and JAXA the Instrument Optical Bench (IOB) finally converged into a trapezoidal shaped structure. The modified IOB structure is depicted in Figure 1, where the volume available to Safari is indicated in green. With the crowded arrangement of the FPI pick-off mirrors near the focal plane at one side and the corner of the trapezoidal IOB at the other side, it became clear that the optical design of Safari would be heavily constrained by available volume inviting for a compact three-dimensional arrangement of the optics. In particular, the existing Phase A1 design was no longer compatible with the changed environment.
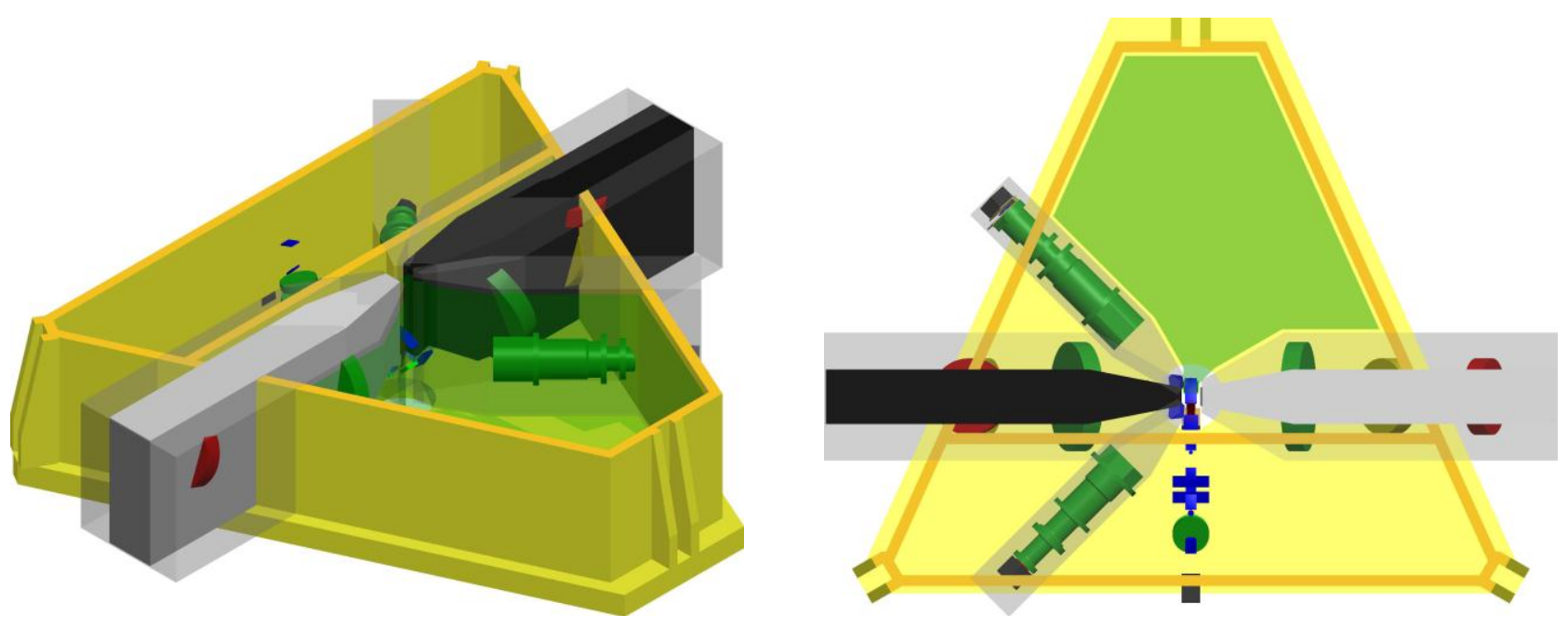

Figure 1. Instrument Optical Bench Configuration. The area indicated in green is reserved for Safari. The yellow structure (box) represents the updated IOB concept. 


\section{DESIGN GOALS AND REQUIREMENTS}

The main optical requirements derived from the science to be carried out with Safari are:

- The instrument shall provide an instantaneous FoV of 2'x2' at the sky, with Nyquist sampled and diffraction limited imaging capability at $\lambda=40 \mu \mathrm{m}$, in three spectral bands covering the $34-210 \mu \mathrm{m}$ wavelength range.

- The instrument shall provide a Fourier-Transform Spectrometer (FTS) spectroscopy mode by means of an optical interferometer configuration yielding a spectral resolving power $\mathrm{R}=2000$ at $\lambda=100 \mu \mathrm{m}$ for each pixel in the science FoV.

- The instrument shall provide an input port coupled to an internal radiometric calibration source.

- The instrument shall provide a filter wheel providing a set of pre-selected band-pass or special filters in each spectral band.

- The instrument shall provide means to control out-of-band and outside FoV straylight.

The key interface requirements applicable to the optical design of the instruments are:

- The instrument shall interface to the SPICA telescope having a focal ratio of f/5.4 and an effective focal length of $16200 \mathrm{~mm}$ (see Table 1) picking off the science FoV at an off-axis position of 7'.

- The instrument shall interface to the Focal Plane Arrays (FPA's) having a focal ratio in the range for f/15 to $\mathrm{f} / 20$.

Finally the following general design goals are applicable:

- The instrument design shall be highly modular with a clean arrangement of the FPA's and cryocooler allowing good access, thermal and electrical interfaces and integration possibilities.

- The design should offer good possibilities to implement light tight comparments sealed by out-of-band straylight filters.

\section{CONCEPTUAL DESIGN DESCRIPTION}

\subsection{Optical sub-systems and instrument topology}

The Safari optical system is divided into three main optical sub-systems:

- $\quad$ The Input Optics Module (IOM)

- The Interferometer or FTS Optics

- The Camera Bay Optics

The overall instrument topology is indicated in Figure 2. At the right hand side the Pick-Off Mirror (POM) can be seen, followed by the IOM. Not shown in the figure is an instrument shutter that will close the entrance aperture to the instrument for dark calibration of the instrument. The IOM internally interfaces to the interferometer optics.

As will be described later the Safari optics features a Mach-Zehnder interferometer configuration, which is composed of two layers indicated in the figure as the MZ-1 and -2 layers. The FTS mechanism modulating the optical pathlength in the interferometer arms is indicated by FTSM. The design of the FTSM is made by TNO and described in [8].

The Camera bay optics is finally composed of a so-called (cold) detector box, providing an environment of low thermal background $(1.7 \mathrm{~K})$ and a cold pupil stop for the long-wave channel. The detector box contains optics relaying the output ports of the Mach-Zehnder interferometer to the three FPA's. The three FPA's divide the Safari spectral range into three octave bandwidth detector bands. The FPA's contain the large-format TES arrays operated at $50 \mathrm{mK}$ and provide the necessary thermal and magnetic shielding[3]. Finally the cryocooler [9-10] for the TES arrays is indicated at the back of the instrument. 


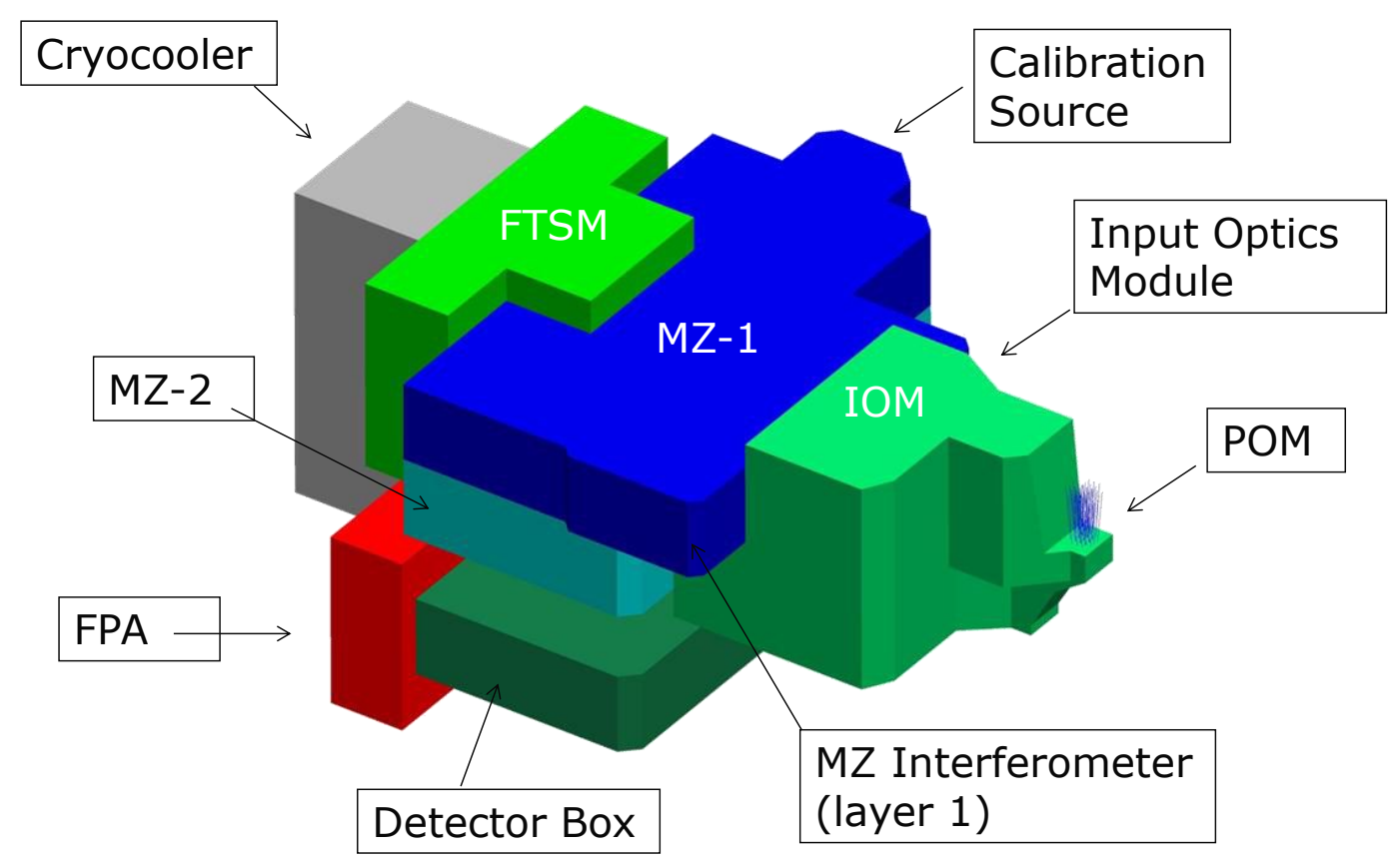

Figure 2. The Safari instrument topology indicating the optical subsystems and units.

A block diagram of the optics inside the optical subsystem is finally shown in Figure 3 for later reference. In the block diagram the powered mirrors are labeled by "M", whereas the folding mirrors are labeled by "FM". All powered mirrors are conical. In the following three subsections some design details of each of the optical subsystems will be provided.

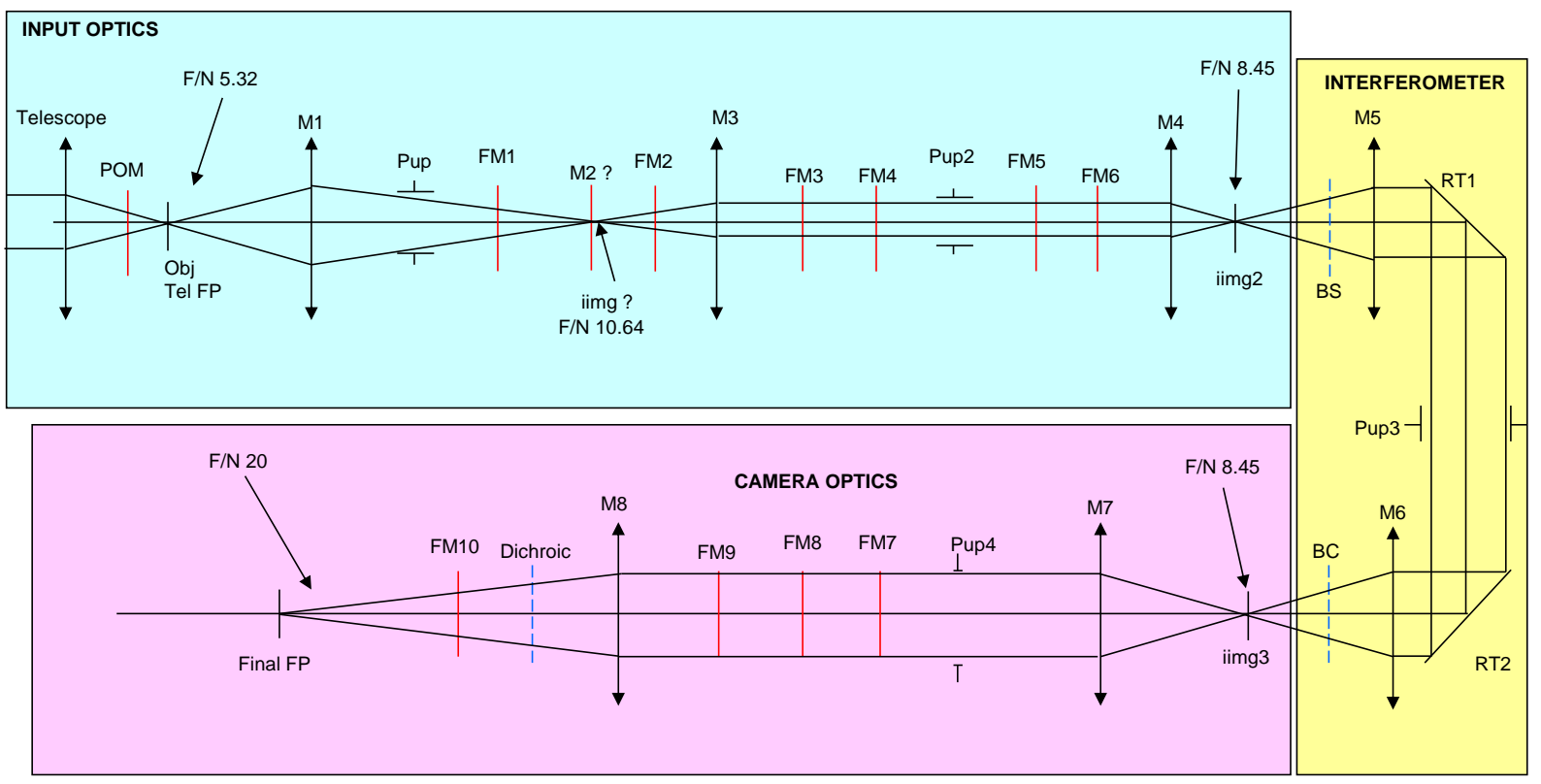

Figure 3. Optical block diagram of the Safari optical design. 


\subsection{The Input Optics Module (IOM)}

The IOM contains 11 mirrors including the POM. It contains 3 powered mirrors, the rest of the optics are folding mirrors required to keep the overall volume of the instrument as compact as possible. The telescope beam is picked up before the focal plane image which provides internal access to the focal plane. The IOM provides two internal field images as well as two pupil images. At the first field image a field limiting aperture will be placed. There is provision for a second field aperture at field mirror M2 which can be used for straylight control. The first pupil image is formed after M1 and a second pupil image is available in between folding mirror FM4 and FM5. The IOM form a relay with magnification of about 1.6 re-imaging the input focal plane with focal ratio $\mathrm{f} / 5.4$ to an output image in front of the interferometer optics with a focal ratio of about f/8.5. In Figure 4 the optics are shown in conjunction with the instrument topology.

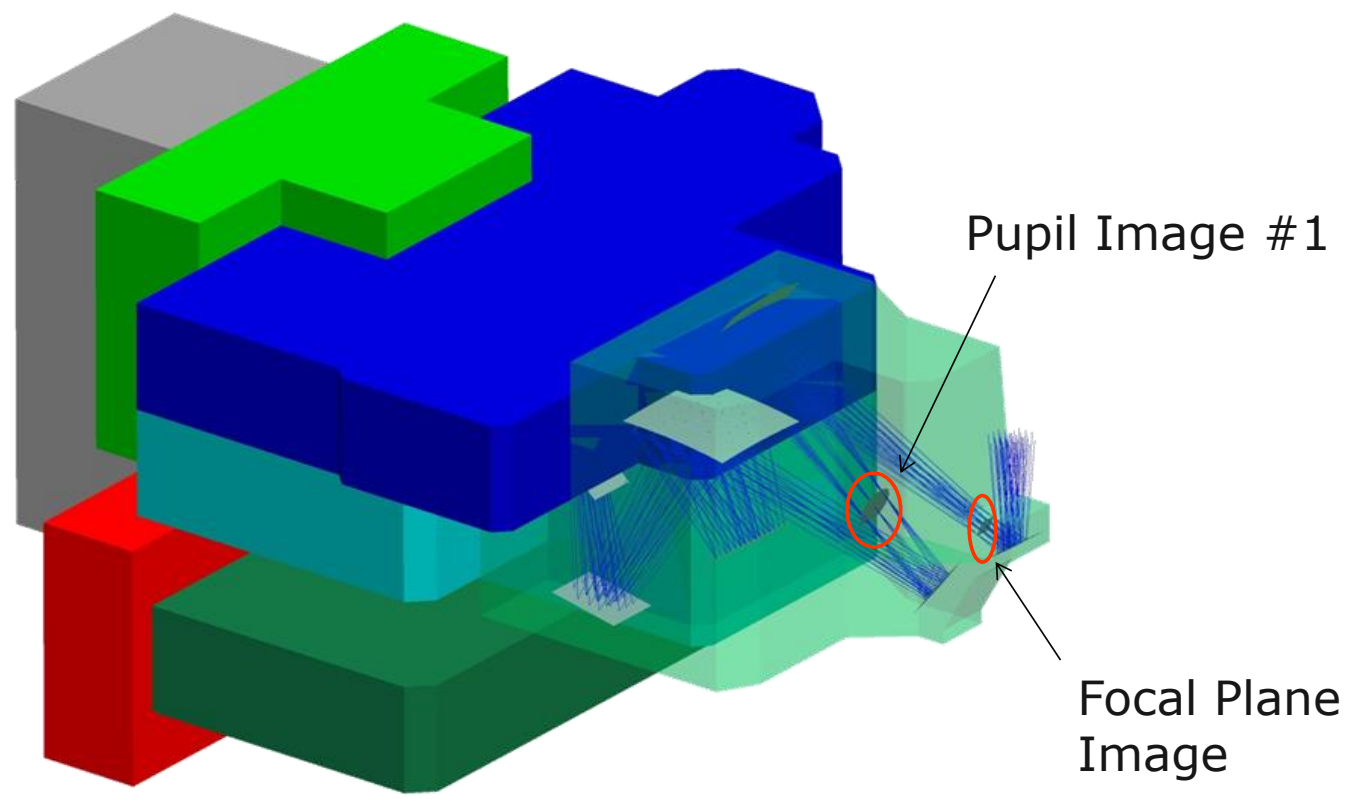

Figure 4. IOM optics showing the internal focal plane image and first pupil image.

\subsection{The interferometer optics}

The interferometer optics accept the f/8.5 beam from the IOM which is collimated by M3 forming a pupil image at the centre of the rooftop mirror configuration of the FTS mechanism. The interferometer optics are symmetric in the pupil of the FTSM for zero Optical Path Difference (OPD) and divided up into two compartments forming two layers of the overall optical assembly. The interferometer optics are made up of 4 curved mirrors (2xM5 and 2xM6) and contain the beam-splitter and -combiner optical elements.

In Figure 5 the bottom layer of the interferometer optics is shown (MZ-1). From the right the light collected at the POM and passed by the IOM enters the interferometer at an intermediate field image. The mirrors M5 at the bottom left and right collimate the light into the direction of the RF mirrors on the FTS mechanisms which passes the light to a second symmetric layer of optics ending with the beam-combiner as is shown in Figure 6. At the junction of the two levels of the interferometer optics a pupil plane is located in between the two separate mirrors forming an effective rooftop mirror.

\subsection{The camera bay optics}

The final part of the optical train is formed by the camera bay optics which starts at a field image in the output of the interferometer. The LW band is located in one output port, whereas the SW/MW bands are in the other port. Each output port has its own dedicated filter wheel, meaning that 9 positions are available for the combined SW/MW band and 9 
independent positions for the LW band. The position of the filter wheels driven by their pre-defined unit envelope is illustrated in Figure 7. The outer envelope of the filter wheels has driven the filter wheels into a peculiar position of the instrument where they are still accessible but force the input optics to become rather complicated. It is clear from Figure 7 that the location of the filter wheels requires the IOM to fold and wrap the telescope beam around the central part of the instrument containing the filter wheels explaining the relatively large amount of folding mirrors required in the IOM. On a positive note the IOM has become rather flexible and still compact and provides good handles for future beam and straylight control measures. The filter wheels are positioned in the vicinity of a pupil image (clearly visible in Figure 6) which sits at the entrance of the cold detector box. This pupil image is formed by a powered mirror M7 following the image plane of the interferometer.

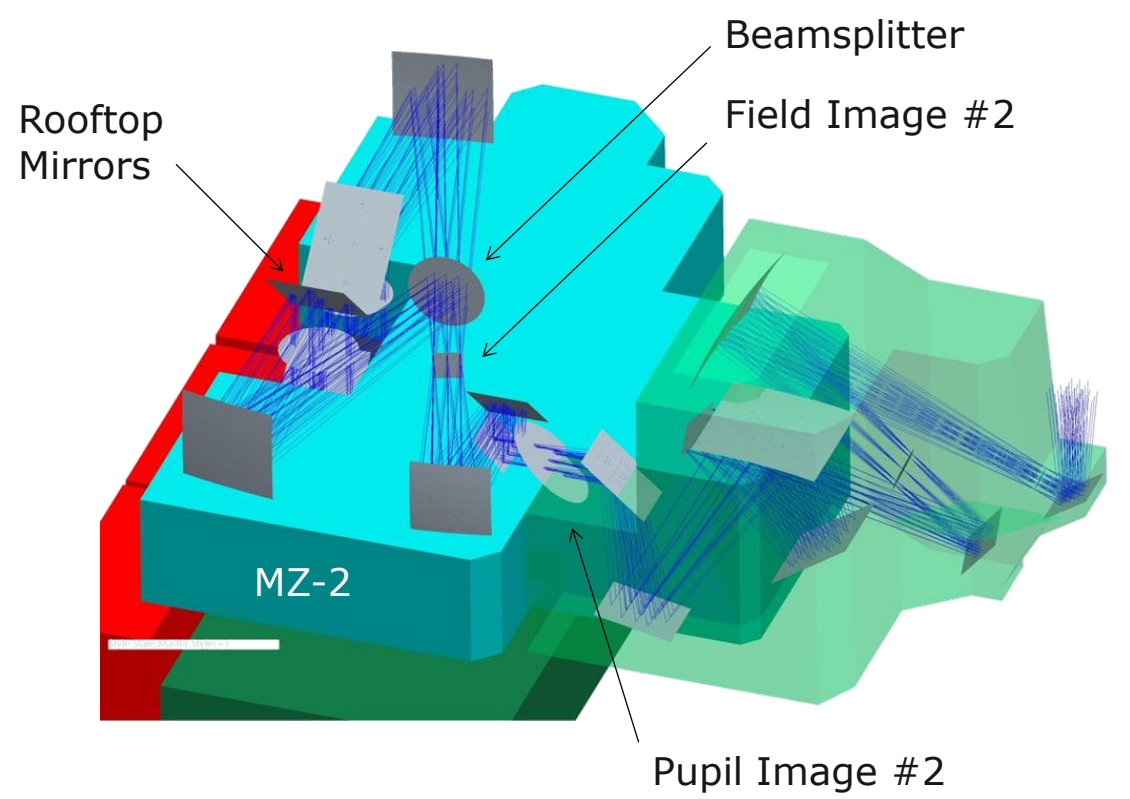

Figure 5. Bottom layer of the Safari interferometer optics.

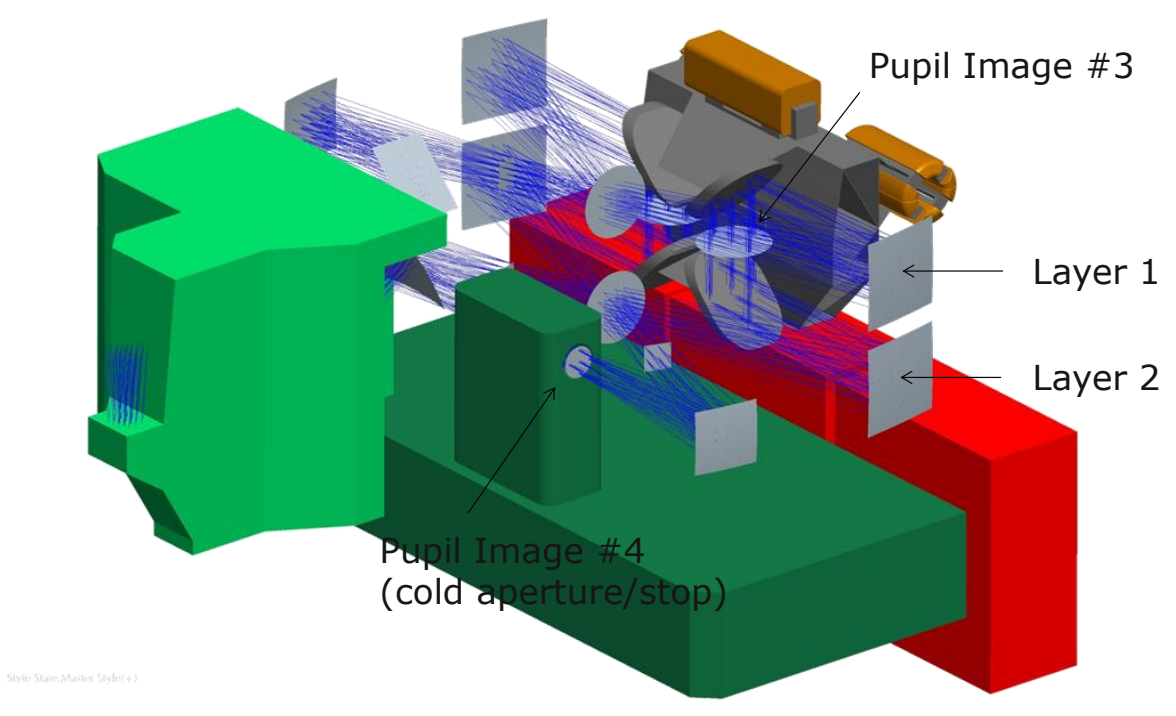

Figure 6 - Second layer of the interferometer optics of Safari. 


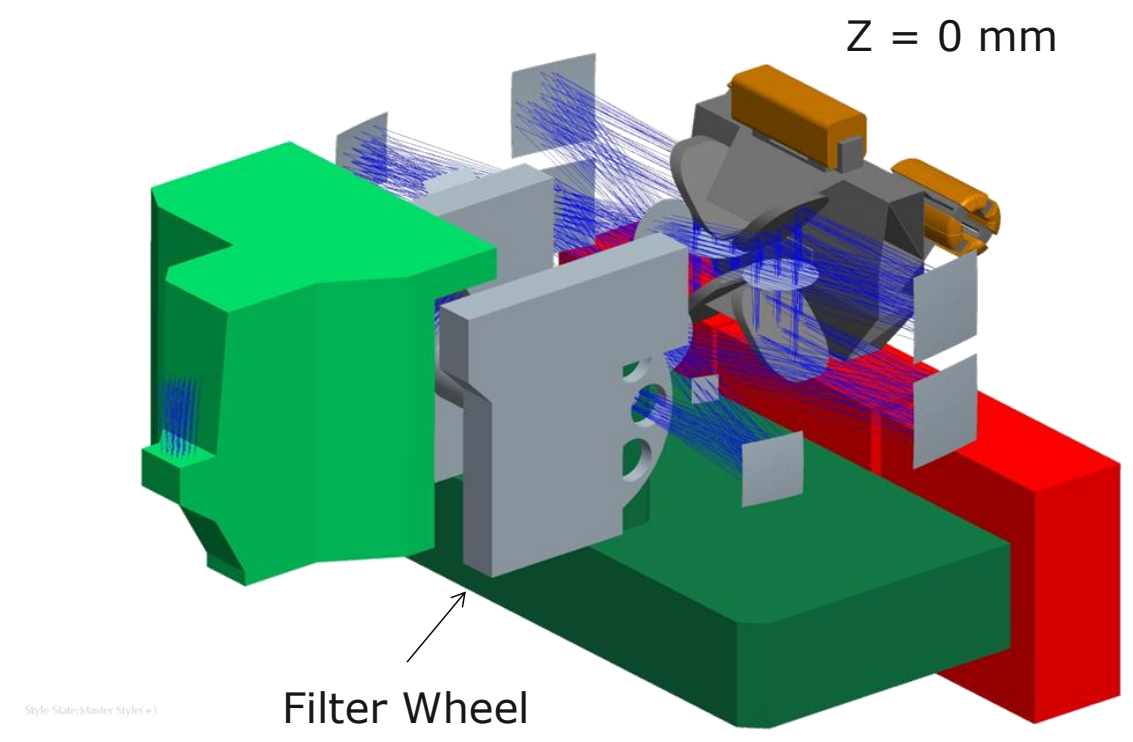

Figure 7. Illustration of the central position of the filter wheels.

Inside the detector box there are mainly folding mirrors and for each band one powered mirror M8 providing the $\mathrm{f} / 15$ to $\mathrm{f} / 20$ interface range for the FPA's. The situation is illustrated in Figure 8, which together with Figure 6 provides a good overview of the camera bay optics. In between the pupil plane forming the interface to the detector box and the final beam-forming mirror M8 there are in total 3 folding mirrors in each band. The dichroic in the SW/MW band, reflecting the short wavelength radiation, is used at an angle of incidence below $22.5^{\circ}$. After each beam forming mirrors there is one final folding mirror in each band allowing for a compact, fair and square arrangement of the three FPA's next to each other with good access to the interface plane of the FPA's. The camera bay optics form a virtual exit pupil, the only physical pupil plane is therefore at the entrance of the cold detector box.

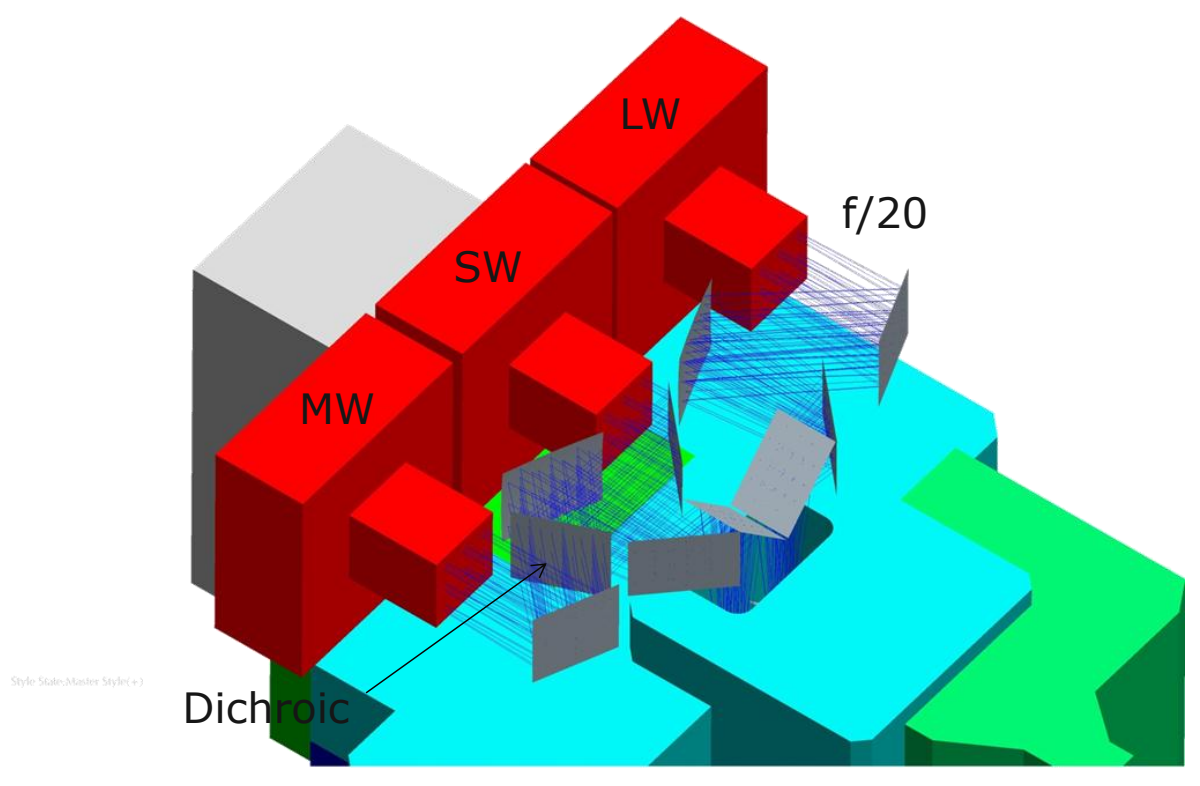

Figure 8. Open box view on the camera bay optics contained in the cold detector box. 


\section{FUTURE WORK}

The optical design presented in this paper was originally designed by TNO Optics in close collaboration with the Safari optical working group. The design offers a principle solution that is compliant with the main requirements and external boundary conditions. Further work is however required to obtain a better definition of the calibration source, filter wheel and shutter mechanism in and near the IOM. Currently conceptual design work with consortium partners is ongoing in all three areas in order to obtain solid and compliant designs with clear indications of the required volume[11]. Once these concepts have matured, the Safari optical working group will undertake a joint effort to converge to a final optical design concept. Meanwhile an alternative design for a more compact IOM has been completed by INTA, anticipating on the expected volume requirements of the shutter, filter wheel and calibration source. Following up current design iterations of the FPA's, INTA has also prepared a different L-shaped arrangement of the FPA's in the camera bay optics. After final modification of the TNO design by a joint effort of the optical working group it is currently foreseen that CAB-INTA will take the lead in detailed opto-mechanical design and manufacturing of the optical system in Phase B and beyond.

\section{SUMMARY AND CONCLUSION}

In this paper the current optical design concept of the Safari instrument on SPICA has been presented. A consistent optical end-to-end design has been described, satisfying the key optical requirements and external boundary conditions. A major challenge for the optical design is to comply with relatively little available volume for the instrument without compromising the optical performance. The results presented in this paper illustrate that this can be accomplished, although final optimization of the design and a detailed performance analysis is still pending on the final conceptual definition of several sub-units of the instrument. In terms of accommodating all optical functions and satisfying the main interfaces a fully compliant design concept has been achieved.

\section{REFERENCES}

[1] B. Swinyard, T. Nakagawa, et al, "The space infrared telescope for cosmology and astrophysics: SPICA A joint mission between JAXA and ESA", Experimental Astronomy, vol. 23, pp. 193-219 (2009).

[2] P. Roelfsema, et al, "The Safari imaging spectrometer for the SPICA space observatory", to appear in these proceedings.

[3] B. D. Jackson, et al, "The SPICA-Safari detector system: TES detector arrays with frequency division multiplexed SQUID readout", IEEE Transactions on Terahertz Science and Technology, Vol. 2, pp. 12 (2012).

[4] K. Dohlen, A. Origné, D. Pouliquen, and B. Swinyard, "Optical design of the SPIRE instrument for FIRST", Proc. SPIE 4013, Munich, 27-31 March (2000).

[5] B. M. Swinyard, et al, "The FIRST-SPIRE spectrometer a novel imaging FTS for the sub-millimetre", Proc. SPIE 4013, Munich, 27-31 March (2000).

[6] T.C. van den Dool, et al, "The development of a breadboard cryogenic optical delay line for Darwin", Proc. SPIE 6692, pp. 66920A.1-66920A.12 (2007).

[7] T.C. van den Dool, et al, "Cryogenic magnetic bearing scanning mechanism design for the SPICA/SAFARI Fourier Transform Spectrometer", Proc. SPIE 7739 (2010)

[8] T.C. van den Dool, et al, "SPICA/SAFARI Fourier Transform Spectrometer Mechanism Evolutionary design", to appear in these proceedings.

[9] L. Duband, J.M. Duval, N. Luchier and T. Prouve, Cryogenics 2012, Vol. 52, pp. 145-151.

[10] I. Charles, et al, "Thermal architecture of the SPICA/SAFARI instrument", to appear in these proceedings.

[11] M. Eigenmann, U. J. Wehmeier, A. Vuilleumier, G. Messina, M. R. Meyer, "Conceptual Phase A design of a cryogenic shutter mechanism for the Safari flight instrument", to appear in these proceedings. 